\section{Mid-term follow-up after heart valve replacement with CarboMedics bileaflet prostheses}

\section{To the Editor:}

I read with interest the article by de Luca and associates $^{1}$ concerning valve replacement with the CarboMedics bileaflet prosthesis, and I commend them for providing valuable information to help surgeons evaluate the recently approved CarboMedics valve (CarboMedics, Inc., Austin, Tex.). The authors have carefully adhered to the published guidelines for reporting valve-related complications $^{2}$ in virtually all areas. The completeness of their follow-up and the detail of their presentation are admirable.

One area of their report has results that appear to be misleading, namely, the presentation of linearized rates of valve-related complications reported for aortic, mitral, and double valve replacement. The presentation of accurate information is important, because on page 1163 of their report the authors state that their incidence of thromboembolic complications is low, at $0.5 \% / \mathrm{pt}-\mathrm{yr}$, compared with other series. Analysis of the information provided in the Results section on page 1160 reveals that the linearized rates calculated for the individual events are probably erroneous. For example, the authors state on page 1160 that thromboembolic events were observed in seven patients. If the cumulative patient follow-up was 478 patient-years, as stated on page 1159 , then the linearized rate for thromboembolism would be $1.5 \% / \mathrm{pt}-\mathrm{yr}$, not $0.5 \%$ / pt-yr.

A further difficulty arises when the authors present linearized rates segregated according to valve procedure. Although they do not provide us with a breakdown of the individual events by operative procedure, their report of the linearized rates of thromboembolism for aortic $(0.35 \% / \mathrm{pt}-\mathrm{yr})$, mitral $(0.8 \% / \mathrm{pt}-\mathrm{yr})$, and double valve replacement $(0.3 \% / \mathrm{pt}-\mathrm{yr})$ cannot be correct. Linearized rates for valve-related complications separated according to valve operation must be divided by the patient-years of follow-up for only that valve operation group to accurately represent the linearized rate. The authors appear to have generated a "linearized rate" by dividing the percentage of patients free of an event actuarially by the follow-up interval of $3 \frac{1}{2}$ years.

Similar computational errors can be found in the presentations of valve-related death, anticoagulant-related hemorrhage, nonstructural valvular dysfunction, prosthetic valve endocarditis, and incidence of reoperation. In terms of sustaining the high quality of their report, I ask that de Luca and colleagues generate accurate linearized rates for the valve-related complications that they have noted for the combined and separate operative procedures. This information would be valuable for cardiac surgeons who are seeking to generate data ${ }^{3}$ that allow comparison of the various available prostheses, with an aim toward finding the most appropriate prostheses for their patients.

Cary W. Akins, MD Department of Cardiac Surgeny Massachusetts General Hospital Boston, MA 02114

\section{REFERENCES}

1. de Luca D, Vitale N, Giannolo B, et al. Mid-term follow-up after heart valve replacement with CarboMedics bileaflet prostheses. J Thorac CardiovasC SURG 1993;106:1158-65.

2. Edmunds LH, Clark RE, Cohn LH, et al. Guidelines for reporting morbidity and mortality after cardiac valvular operations. J THORAC CARDIOvASC SURG 1988; 96:351-3.

3. Akins CW. Mechanical cardiac valvular prostheses. Ann Thorac Surg 1991;52:161-72.

$12 / 8 / 65675$

\section{Reply to the Editor:}

We appreciate the opportunity to respond to the comments of Dr. Akins regarding our article. ${ }^{1} \mathrm{He}$ gave us the benefit of his experience. ${ }^{2}$

With regard to linearized rates of valve-related complications, data were analyzed by applying the MantelHaenszel test included in the program 1L of the BMDP statistical software package (BMDP Statistical Software, Inc., Los Angeles, Calif.). ${ }^{3}$ This test takes into account the number of events for a given time divided by the number of patients followed up for the same time interval. ${ }^{3,4}$ In our study we assumed a 6-month interval. This type of calculation is presented in our article as the curve at the bottom of each graph. The combined and separate linearized rates presented in our report were obtained with this test, by summing up the patient-year incidences of each time interval divided by the total number of time intervals of follow-up. ${ }^{3,4}$ Moreover, linearized rates of each subgroup of patients were calculated including only the patients undergoing that operative procedure. For all these reasons, they would be better defined as linearized incidences than as linearized rates, and we apologize not to have mentioned this statistical aspect in our paper.

On the other hand, linearized rates are generated by the number of events divided by the number of patient-years. As requested by Dr. Akins, we list the linearized rates of our population for the valve-related complications that we have noted for the combined and separate operative procedures (Table I). The rates were calculated according to this latter method.

We are grateful to Dr. Akins for raising this statistical issue of our paper that would have been overlooked

Table I. Linearized rates of valve-related complications

\begin{tabular}{lcccc}
\hline \multicolumn{1}{c}{ Event } & $\begin{array}{c}\text { Total } \\
\text { (\%/pt-yr) }\end{array}$ & $\begin{array}{c}\text { MVR } \\
(\% / p t-y r)\end{array}$ & $\begin{array}{c}A V R \\
(\% / p t-y r)\end{array}$ & $\begin{array}{c}D V R \\
(\% / p t-y r)\end{array}$ \\
\hline NSD & 0.6 & - & 0.5 & 2 \\
ARH & 1 & - & 1.5 & 2 \\
TE & 1.5 & 2.9 & 1 & - \\
Reoperation & 0.6 & 1.7 & - & - \\
PVE & 0.2 & - & 0.5 & - \\
VRD & 1.5 & 1.2 & 1.5 & 2 \\
\hline
\end{tabular}

$A R H$, Anticoagulant-related hemorrhage; $A V R$, aortic valve replacement $D V R$, double (mitral and aortic) valve replacement; $M V R$, mitral valve replacement; $N S D$, nonstructural dysfunction; $P V E$, prosthetic valve endocarditis; $T E$, thromboembolic events; $V R D$, valve-related deaths. 
otherwise. A correct analysis of data on cardiac valvular prostheses allows a better understanding of the results of such procedures and enables surgeons to choose the appropriate prostheses for their patients.

Nicola Vitale, $M D$

Luigi de Luca, MD

Istituto di Cardiochirurgia

Università d Bari

Piazza Giulio 11

70124 Bari, Italy

Maurizio Cotrufo, $M D$

Istituto di Cardiochirurgia

Seconda Università di Napoli

Ospedale $V$. Monaldi

Via L. Banchi 3

80100 Napoli, Italy

\section{REFERENCES}

1. de Luca L, Vitale N, Giannolo B, et al. Mid-term follow-up after heart valve replacement with CarboMedics bileaflet prostheses. J Thorac CardovasC SuRG 1993;106:1158-65.

2. Akins CW. Mechanical cardiac valvular prostheses. Ann Thorac Surg 1991;52:161-72.

3. BMDP Program 1L. BMDP statistical software manual. Vol 2. Los Angeles: University of California Press, 1990.

4. Colton T. Statisties in medicine. Boston: Little Brown, 1978.

$12 / 8 / 65676$

\section{Tracheal sleeve pneumonectomy for bronchogenic carcinoma \\ To the Editor:}

The January 1994 issue of this Journal included our article, titled "Tracheal Sleeve Pneumonectomy for Bronchogenic Carcinoma" (J Thorac Cardiovasc Surg 94; 107:13-8). The work reported our experience with 28 tracheal sleeve pneumonectomies (TSPs), 27 right TSPs and one left TSP. The very low operative mortality (only one death, which occurred on the thirtieth postoperative day as a result of myocardial infarction) and the absence of anastomotic complications (stenosis and dehiscence) seemed to impress the readers, as indicated by the many reprint requests and letters with specific questions on technical details of tracheobronchial anastomosis, on anesthesia modalities, and on preoperative and postoperative care.

High mortality rates are commonly reported in the literature ( $8 \%$ to $29 \%$ ). The progress of ventilation techniques and of suture-performing modalities have allowed acceptance of TSP. Nevertheless, TSP is still carried out only in centers highly qualified to perform thoracic surgery.

From 1992 (when the period of observation ended) to date we have performed seven more right TSPs on patients with lung cancer involving the tracheobronchial angle. In this last group of patients we recorded two deaths caused by postoperative complications which, in our opinion, were due to inappropriate preoperative radiotherapy. In this letter we would like to stress the negative effects of high-dose radiotherapy in patients eligible for TSP.

Like other authors, we adopted preoperative radiation treatment to reduce the tumor size, so as to facilitate the anastomotic procedure. We chose the low-dose protocol introduced by Paulson and associates, ${ }^{1}$ reserving it for large neoplasms that might be expected to benefit most from combined radiotherapeutic and surgical treatment. No radiotherapy is planned in small tumors.

In our series, of 35 patients subjected to TSP, 14 received a preoperative radiotherapy protocol consisting of 10 treatments of 30 Gy each. One patient had preoperative chemotherapy. Another patient had both radiotherapy and chemotherapy. Seventeen patients did not receive any preoperative treatment. Two patients had high-dose radiotherapy (60 Gy). Their clinical history and the reasons for the high-dose radiation treatment, which in our opinion caused the patients' deaths, are briefly described.

The first patient, a 67-year-old man, was counselled to receive radiotherapy in $30 \mathrm{~Gy}$ doses. We were unaware that his son, who is a physician, decided to subject the patient to $60 \mathrm{~Gy}$ doses. Evaluation after radiotherapy showed a good result and the patient was therefore subjected to TSP. The postoperative course was normal until the ninth postoperative day, when clinical and roentgenographic signs of bronchial fistula developed. A bronchoscopic examination showed a hyperemic anastomosis covered with fibrin and without any sign of tissue healing: the endoscopic picture closely resembled that of the immediate postoperative period. A small bronchial fistula was found on the right edge of the suture. A tracheostomy was done and the patient was intubated with the same tube (Sybilla's tube) that we used to carry out the anastomosis, by inflating the balloon distal to the anastomosis. The pleural cavity was drained. The patient had a number of complications, and he died on postoperative day 22 of a fatal hemorrhage from a gastric ulcer. Repeated bronchoscopic studies during the course had all failed to show granulation tissue or other signs of healing on the anastomosis.

The second patient was a 71-year-old man. Despite our prescription for preoperative radiation therapy in $30 \mathrm{~Gy}$ doses, his condition was judged inoperable by the radiotherapist who therefore subjected him to $60 \mathrm{~Gy}$ doses. This treatment yielded a remarkable reduction of the mass. Because we were not sure of the actual responsibility of excessive preoperative radiation in determining bronchial dehiscence in the previous case, and considering the good general conditions of this patient, we decided to perform TSP.

On the first postoperative day a conspicuous bronchorrhea developed that necessitated repeated bronchial suctions. The patient died on the eighth postoperative day of pulmonary edema. A bronchoscopic examination had revealed a normal tracheobronchial anastomosis.

Jensik and coworkers, ${ }^{2}$ in an article published in 1982, had already stressed the fact that postoperative mortality 